\title{
Development of ADN-based Thruster Technologies - An overview of the European Project Rheform
}

\author{
Michele Negri ${ }^{1}$, Marius Wilhelm, Christian Hendrich \\ DLR, German Aerospace Center, Institute of Space Propulsion, Lampoldshausen, Langer Grund, 74239 \\ Hardthausen, Germany \\ Niklas Wingborg, Jonas Johansson, Martin Skarstind \\ FOI, Swedish Defence Research Agency, SE-14725 Tumba, Sweden \\ Corentin Maleix, Romain Beauchet, Yann Batonneau, \\ IC2MP, CNRS-UMR 7285, University of Poitiers, 4 rue Michel Brunet, bâtiment B27, TSA 51106, 86073 Poitiers \\ Cedex 9, France \\ Robert-Jan Koopmans \\ FOTEC Forschungs- und Technologietransfer GmbH, Viktor Kaplan-Straße 2, 2700 Wiener Neustadt, Austria \\ Wilhelm Dingertz, Kjell Anflo \\ ECAPS, Torggatan 1517104 Solna, Sweden \\ Ulrich Gotzig \\ ArianeGroup, Langer Grund, 74239 Hardthausen, Germany \\ Martin Schwentenwein \\ Lithoz GmbH, Mollardgasse 85a/2/64-69, 1060 Vienna, Austria
}

\begin{abstract}
In the European project Rheform research activities aimed at improving ADN-based propulsion systems have been conducted. ADN-based propellants are extremely promising as hydrazine replacement. They have a lower overall life cycle cost due to simplified handling, higher specific impulse and higher density compared to hydrazine. In the paper an overview of the main results achieved during the project will be given.
\end{abstract}

\section{Nomenclature}

$\begin{array}{ll}\text { ADN } & \text { Ammonium dinitramide } \\ \text { AOCS } & \text { Attitude and Orbit Control System } \\ \text { RACS } & \text { Roll and Attitude Control System } \\ \text { I }_{\text {sp }} & \text { Specific Impulse }\end{array}$

\section{Introduction}

Hydrazine is the standard monopropellant for spacecraft propulsion system since the 1960s, but it is highly toxic and carcinogenic. Therefore it requires extensive safety procedures during each phase of its use (i.e. testing, shipping, tanking, in-flight operations and waste disposal). Such complexity is associated with a high life cycle costs, even if hydrazine itself is not expensive. The reduction of life cycle cost is one of the main drivers behind developing hydrazine replacements. Moreover, new regulations may lead to restriction of their use in the near to mid-term. For example, in Europe hydrazine has been added to the list of substances of very high concern from the authority in charge of regulating the use of chemicals (ECHA). The substitution of hydrazine with green propellants may lead to significant benefits, if suitable propellants are selected. Very interesting replacements for hydrazine are liquid propellants based on ammonium dinitramide $\left(\mathrm{ADN}, \mathrm{NH}_{4}{ }^{+} \mathrm{N}\left(\mathrm{NO}_{2}\right)_{2}{ }^{-}\right)$. They offer the following advantages compared to hydrazine:

\footnotetext{
${ }^{1}$ Corresponding author: michele.negri@dlr.de
} 
- Simplified handling, especially loading at the launch site. During the PRISMA mission, the first mission in which a ADN-based thruster was demonstrated in space, the man-hours for loading hydrazine were three times more than for LMP-103S [1].

- Higher overall performance $\left(\mathrm{I}_{\mathrm{sp}}\right)$. In the PRISMA mission the specific impulse was 6 to $12 \%$ higher compared to hydrazine [2].

- Higher volumetric specific (about 32 \% more) impulse due to higher density leading to smaller tanks, and therefore reduced structural weight [2].

To develop new technologies for improving ADN-based propulsion systems, the Rheform project was started. Rheform is a project funded from the European's Union Horizon 2020 programme. The name Rheform stands for: "Replacement of hydrazine for orbital and launcher propulsion systems". The project ran from January 2015 to the end of 2017. The Rheform consortium comprises 9 entities from 4 European countries: Austria, France, Germany and Sweden. Two universities are involved: the University of Poitiers (UP and the University of Applied Sciences Wiener Neustadt (FHWN). Three research centers are participating to the project: the German Aerospace Center (DLR), the Swedish Defence Research Agency (FOI), and the French National Center for Scientific Research (CNRS). Two small companies are involved: Lithoz and FOTEC. Finally two space companies are participating: ECAPS and ArianeGroup (AG).

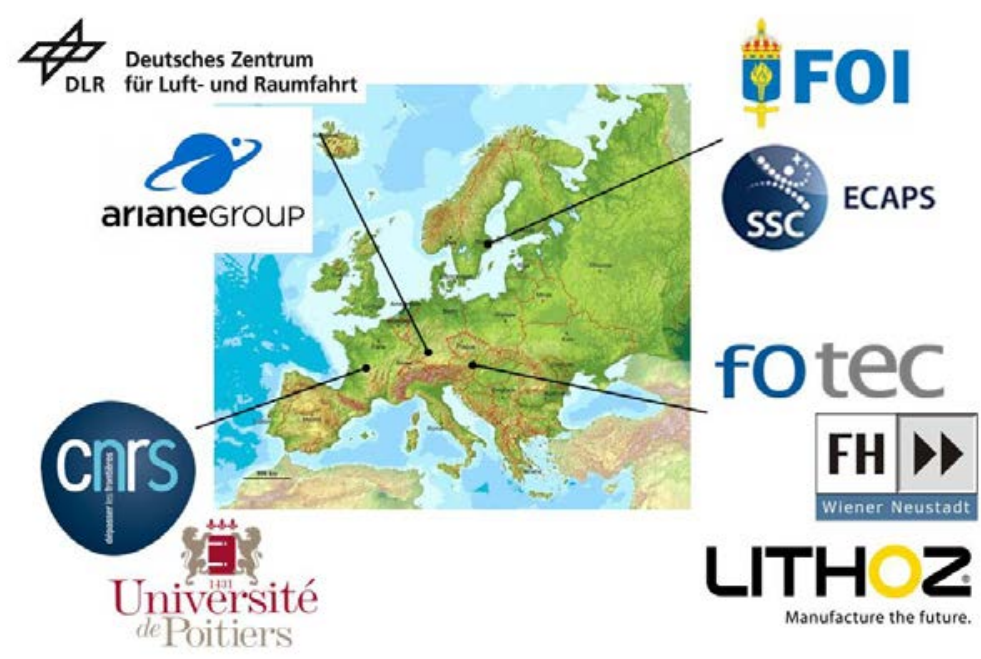

Fig. 1 Rheform partners and their locations in Europe.

\section{Selection of two reference cases}

The selection of two trust classes and corresponding reference cases was one of the first activities conducted. The most urgent need of green thrusters was seen in the $20 \mathrm{~N}$ class for Attitude and Orbit Control (AOCS) and in the 200 $\mathrm{N}$ class for Roll and Attitude Control (RACS) and Deorbiting. A $1 \mathrm{~N}$ green thruster was already available and commercialized by the project partner ECAPS. $20 \mathrm{~N}$ hydrazine thrusters had a larger market share compared to $10 \mathrm{~N}$ ones. Moreover $20 \mathrm{~N}$ thrusters could also be used for deorbiting whereas $10 \mathrm{~N}$ does not provide enough thrust. Above $400 \mathrm{~N}$ the need for a thruster was small.

The Planck scientific satellite has been taken as reference case for the $20 \mathrm{~N}$ thrusters. The AOCS of such satellite is equipped with twelve $20 \mathrm{~N}$ hydrazine monopropellant thruster. The RACS of the Vega launcher has been taken as reference case for the $200 \mathrm{~N}$ thruster. Such system is equipped with six $200 \mathrm{~N}$ hydrazine thrusters.

\section{Adapting the composition of the propellant to enable the use of cheaper materials for the combustion chamber}

A work on the influence of variations in the composition of the two baseline propellants LMP-103S and FLP-106 was conducted. Theoretical calculations have been conducted in order to predict the influence of increased water 
contents in the two baseline propellants (LMP-103S and FLP-106) on the combustion temperatures and on the performance. The amounts of water necessary to obtain combustion temperatures compatible with several potential chamber construction materials were determined and are shown in Table 1.

Table 1: Combustion chambers materials and corresponding maximum admissible temperatures. For each material the propellant composition necessary to obtain a compatible combustion temperature has been determined as well as the corresponding specific impulse.

\begin{tabular}{|c|c|c|c|c|}
\hline \multirow[t]{2}{*}{$\begin{array}{c}\text { Combustion Chamber } \\
\text { Material }\end{array}$} & $\begin{array}{l}\text { Max admissible } \\
\text { temperature }\end{array}$ & Propellant composition & $\begin{array}{l}\text { Calculated } \\
\text { combustion } \\
\text { temperature }\end{array}$ & $\begin{array}{c}\text { Calculated } \\
\text { specific } \\
\text { impulse }^{2}\end{array}$ \\
\hline & {$\left[{ }^{\circ} \mathrm{C}\right]$} & & {$\left[{ }^{\circ} \mathrm{C}\right]$} & [s] \\
\hline \multirow[t]{2}{*}{ Super Alloy } & 1250 & LMP-103S + 17.4\% H2O & 1253 & 226 \\
\hline & & FLP-106 + 27.7\% H2O & 1249 & 217 \\
\hline \multirow[t]{2}{*}{ Platinum Rhodium } & 1500 & LMP-103S + 5.8\% H2O & 1499 & 244 \\
\hline & & FLP-106 + 15.7\% H2O & 1500 & 234 \\
\hline \multirow[t]{2}{*}{ Platinum Iridium } & 1600 & LMP-103S + 1.8\% H2O & 1598 & 251 \\
\hline & & FLP-106 + 11.5\% H2O & 1601 & 241 \\
\hline Ceramic & 1650 & LMP-103 & 1645 & 254 \\
\hline Ceramic & 1900 & FLP-106 & 1904 & 258 \\
\hline
\end{tabular}

More details on how the performances change by varying the propellant composition are given in [3].

\section{Improving the ignition methods}

One of the main goals of Rheform was to improve the ignition methods. ADN-based thrusters are currently ignited with a pre-heated catalyst. The $1 \mathrm{~N}$ thruster from ECAPS features a $10 \mathrm{~W}$ heater. The pre-heating time is 30 minutes. In the case of the PRISMA thruster the maximum load during preheating was 9.25W and 8.3W during firing [4]. Cold start is not possible: the decomposition starts only if the catalyst has reached its operational temperature of $350{ }^{\circ} \mathrm{C}$. This is a limitation of ADN thrusters compared to hydrazine ones: the catalysts currently used for hydrazine ( $\mathrm{S} 405$ or similar) are cold start capable, even if preheating is often used to increase the lifetime of the catalyst. Cold start capability could be important if the thruster has to be used in emergency situation, where there is no time to pre-heat it. A reduction in preheat power would also be a benefit for small satellites, where the available power is limited [4].

The preheating power for larger hydrazine thrusters remains limited to some tenths of Watts. For example, the preheating power for the Aerojet $440 \mathrm{~N}$ thruster is $13.1 \mathrm{~W}$ [5]. On the other hand the preheating power requirements for ADN catalysts increase strongly for larger thruster. This is due to the fact that most of the power is used to evaporate the propellant and the propellant mass flow rate increase nearly linearly with the thrust.

Due to these limitations, the possibility to develop an improved igniter for ADN propellants has been studied in Rheform. In the project both catalytic and thermal igniters have been considered

\section{A. Improving catalytic ignition}

Activities have been conducted in Rheform to develop better catalysts, which require a lower pre-heating temperature. The research has been conducted in parallel both on catalyst using conventional granulated supports and on catalyst based on novel 3D printed monolithic catalyst supports.

The main focus of the work conducted in the first half of the project was on developing very reactive catalysts in order to decrease the amount of pre-heating (see [3]). Some of the active phases tested in a batch reactor reduced the ignition temperature just above $100^{\circ} \mathrm{C}$. In particular pellets made of silicon-doped alumina with an active phase of $\mathrm{Pt}-\mathrm{Cu}$ proved the most active of the catalysts tested with both propellants LMP-103S and FLP-106. All the tests conducted showed that a vaporization of the water content is necessary before the decomposition can start. Therefore the objective of developing a cold-start capable catalytic system was deemed not achievable. Nevertheless

\footnotetext{
${ }^{2}$ The specific impulse has been calculated assuming $\mathrm{P}_{\mathrm{c}}=10 \mathrm{bar}, \varepsilon=40$, expansion frozen at the throat and in vacuum.
} 
some of the newly developed catalyst may have a lower pre-heating temperature, leading to a reduced energy required for pre-heating.

In the second half of the project the focus shifted towards developing catalysts that can survive the conditions in the combustion chamber. The combustion of the two baseline propellants generates extremely harsh conditions for the catalyst: the adiabatic combustion temperature is $1645{ }^{\circ} \mathrm{C}$ for LMP-103S and $1900{ }^{\circ} \mathrm{C}$ for FLP-106 and the decomposition of these propellants generates very reactive species. Previous results had indicated that this is a challenging issue as some of the catalysts selected in the batch reactor may not survive in a thruster.

To develop a catalyst support that may survive in a combustion chamber, a considerable amount of effort was invested in developing hexaaluminates. Hexaaluminates are attractive as catalyst supports and potentially as catalysts because they offer excellent resistance and stability to thermal shocks and high temperatures. Moreover they can be synthesised so that they have a large specific surface area, making them suitable to be used as catalyst support also without a washcoat layer.

The procedures to synthesise and shape-form (pellets, granules) the following hexaaluminates have been developed:

- Barium hexaaluminate $\left(\mathrm{BaAl}_{12} \mathrm{O}_{19}\right)$.

- Modified Ba-Ir-Co hexaaluminate. It consists of barium hexaaluminate $\left(\mathrm{BaAl}_{12} \mathrm{O}_{19}\right)$ after replacement of one mole of the twelve moles of aluminium by one half mole of iridium (Ir) and one half mole of cobalt (Co). Hence the name "Ba-Ir-Co hexaaluminate" of the compound. The introduction of Co and Ir atoms within the barium-aluminium lattice of barium hexaaluminate is attractive as this material acts both as catalyst support and active phase, without needing a further impregnation step.

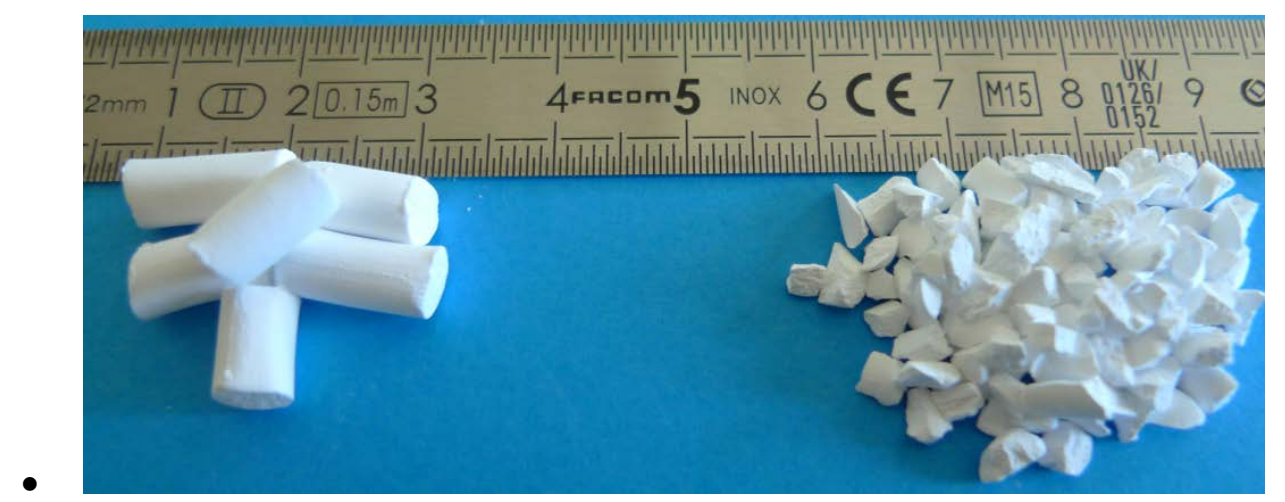

Fig. 2 Barium hexaluminates pellets before (left) and after (right) randomisation.

Once the hexaaluminate powders were synthetized, the pellets were prepared in 4 steps:

- Preparation of a paste. The hexaaluminate powder was mixed with two solvents.

- Extrusion of the resulting paste through a ram extruder to form filaments with a diameter of $5 \mathrm{~mm}$. The filaments were then cut to form small cylinder with a length between 5 and $10 \mathrm{~mm}$.

- Thermal treatment of the pellets. The cylinders were calcinated under air at $600{ }^{\circ} \mathrm{C}$ for $2 \mathrm{~h}$.

- Randomization. The cylinders were broken in smaller pieces with a mortar and sieved to obtain granules with a size between 2 and $2.5 \mathrm{~mm}$.

A novelty introduced by Rheform was the introduction of monolithic catalysts for the ignition of ADN-based propellants. Three different designs were chosen for the printed monoliths: Straight channels (in order to have a direct comparison with an extruded part), cellular structure, and polyhedral structure. For the manufacturing of monoliths 4 different ceramic materials are being evaluated, cordierite $\left((\mathrm{Mg}, \mathrm{Fe})_{2} \mathrm{Al}_{4} \mathrm{Si}_{5} \mathrm{O}_{18}\right)$, aluminium oxide $\left(\mathrm{Al}_{2} \mathrm{O}_{3}\right.$, alumina), magnesium oxide (MgO, magnesia) and silicon nitride $\left(\mathrm{Si}_{3} \mathrm{~N}_{4}\right)$.

In order to verify the survivability of the catalysts (both pellets and monoliths), two different procedures simulating firing conditions on the catalyst were used. A first way to simulate hot firing condition was to conduct hot fire tests using highly concentrated hydrogen peroxide (HTP). The resistance of different monolithic structures to thermal shocks was tested using this procedure. The decomposition temperature of HTP is lower (less than $1000^{\circ} \mathrm{C}$ ) than that of ADN-based propellants, therefore this technique can be used only for a preliminary screening of the catalysts. 
The decomposition and combustion of the baseline propellant LMP-103S creates conditions particularly harsh for the catalyst: the temperature reaches $1600^{\circ} \mathrm{C}$ and one of the main reaction products is water vapor, which could facilitate the sintering of the ceramic support, destroying the mesoporosity and thus dramatically reducing the specific surface area. In order to simulate these conditions, several catalyst samples were tested with a proprietary procedure developed by ECAPS (referred as simulated firing).

The main results of the simulated firing testing are:

- Silicon-doped alumina is not suitable both as granulated support and as washcoat layer for monoliths. The specific surface area (BET) after simulated firing was below measurable level $\left(<0.1 \mathrm{~m}^{2} / \mathrm{g}\right)$.

- $\quad 3 D$ printed magnesia monolith showed some losses in volume (1.7 \%) and mass (0.8\%) after testing.

- 3D printed alumina showed almost no mass loss (0.04\%) and no volume loss. Such catalyst support could be very interesting if a compatible washcoat is found.

- Barium hexaaluminate ( $\mathrm{BaAl}_{12} \mathrm{O}_{19}$, granules) showed some mass loss (1\%) and a sensible loss in specific surface area (75.3\%) but the materials still retained some mesoporosity after the pre-testing.

- Modified Ba-Ir-Co hexaaluminate (granules) showed some mass loss (0.6 \%) and the smallest loss in specific surface area (33\%) of all the material tested in Rheform. The cobalt contained in this material was released in the oven, causing a blue coloration of the sample holder as shown in Fig. 3.

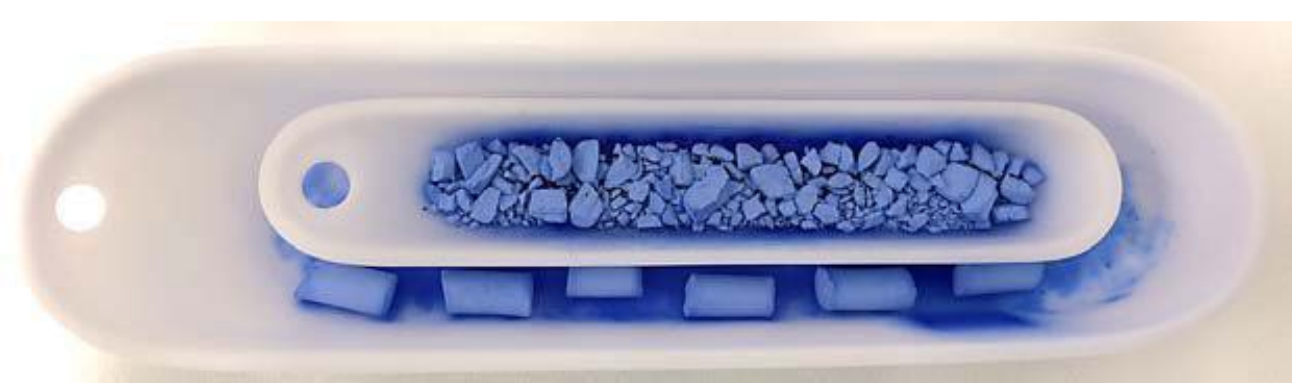

Fig. 3 Hexaaluminates Ba-Ir-Co-Al after pre-testing.

\section{B. Development of a thermal ignition system for the propellant}

In the first half of the project two thermal ignition methods were tested in small scale: laser and resistive ignition, as described in [3]. Both methods were unsuccessful.

The next step was to verify if the two baseline propellants (LMP-103S and FLP-106) could be ignited with a conventional hydrogen/oxygen torch igniter. Such igniter can provide a substantial amount of thermal power (up to $20 \mathrm{~kW}$ ). A torch igniter is not suitable to be used in real application, but tests with this igniter could quantify the amount of energy required for ignition, and if sustained combustion could be achieved once the torch was turned off. To conduct the tests with the torch igniter an experimental setup was designed and realized. A schematic drawing is shown in Fig. 4.

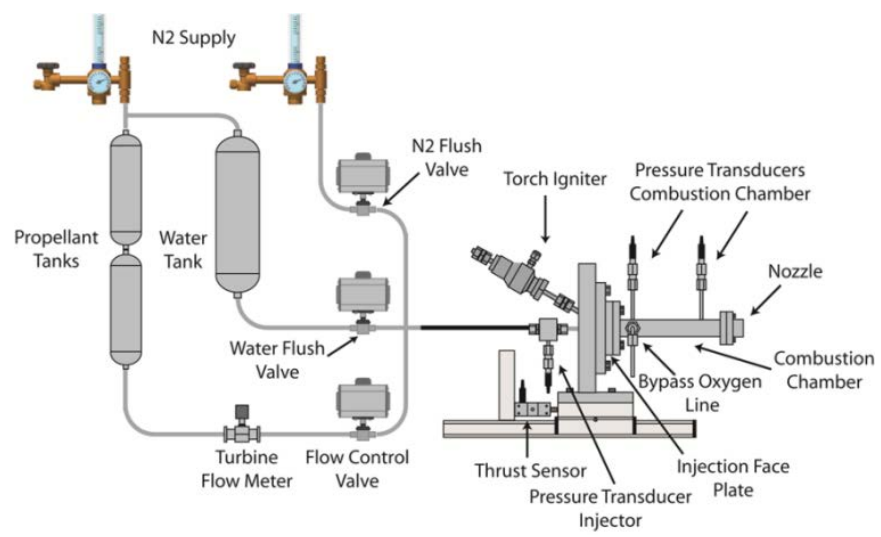

Fig. 4 Schematic drawing of the torch igniter experimental setup. 
The initial tests were conducted with a basic tubular chamber [3]. With this configuration no ignition of the propellants could be achieved. It was assumed that some kind of devices are required in the combustion chamber to facilitate the heat transfer between the hot gasses generated by the torch igniter and the propellant and to increase the propellant residence time in the chamber.

To try and achieve thermal ignition an improvement of the demonstrator design was conducted. Three different designs were so built and tested, called respectively Porous-A, Porous-B, and Porous-C. More than 200 hot fire tests were conducted. The results of this test campaign are described in details in [6], here only a summary of the main results is give.

The idea behind the three designs (Porous A, B and C) was to use a disc of porous material inside a copper inlay. As an example, the configuration Porous $\mathrm{C}$ is shown in Fig. 5. Before propellant injection the copper inlay and the porous material were heated by the torch. This facilitated the vaporization of the propellant, which then came in contact with the hot gasses from the torch already in gas form, so facilitating ignition. The inlay facilitated the heat feedback from the combustion area to the porous material.
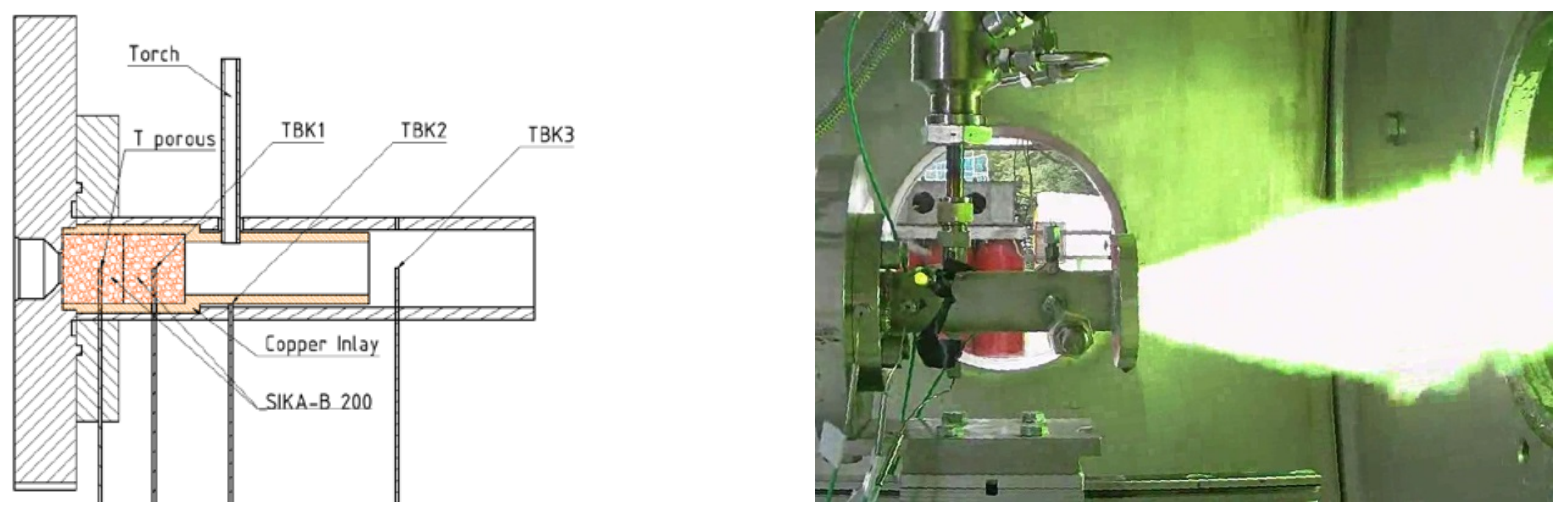

Fig. 5 Setup Porous-C. [Left] Schematic Drawing. [Right] Hot firing test.

The tests with the configuration Porous-C clearly indicated that combustion of the propellant can be achieved when the vaporized propellant is exposed to an ignition source, in this case the hot gasses generated from the torch igniter. No sustained combustion was obtained, i.e. soon after the shutdown of the torch the combustion stopped. This indicated that the heat feedback from the flame to the propellant was not sufficient.

Considering the tests conducted with all three configurations (Porous A, B and C) the following remarks can be made. Thermal ignition of both LMP-103S and FLP-106 was achieved in an open combustion chamber (without nozzle). The propellants burned with a green flame. The color of the flame came probably from the copper inlay and the bronze porous material. The tests conducted clearly showed that a flame holding device facilitates the ignition of the propellants. The effects of the porous material are:

- to store thermal energy to vaporize the propellant

- To increase the residence time of the propellant in the combustion chamber, so increasing the chances of achieving complete vaporization and ignition.

\section{Improvement of the production process of ADN}

In the Rheform project a task was dedicated at improving the production process of ADN. The main producer of ADN for the European market is EURENCO Bofors in Sweden. The small-scale production is performed in a plant initially built for the production of other energetic materials and thus not optimized for the production ADN. As a consequence, $\mathrm{ADN}$ is today very expensive. In order to reduce the cost, ways to improve the synthesis of ADN were studied both in Rheform and in the H2020 project GRAIL [7]. In Rheform the focus was on continues nitration of ammonium sulfamate to guanylurea dinitramide (FOX-12), while in GRAIL a one-step method to convert FOX-12 to ADN was studied.

A pilot plant was designed and built to demonstrate the possibility of a continuous nitration. A photo of the setup is shown in Fig. 6 [Left]. The core of the setup is the continuous flow plate reactor, shown in Fig. 6 [Right]. A substantial amount of effort was required to minimize the leakage between the plates composing the reactor. 
The addition of reaction components, and especially the addition of ammonium sulphamate proved challenging. The work conducted showed that it is possible to form a slurry of ammonium sulphamate in sulphuric acid at room temperature, cooling this slurry, and by addition of nitric acid, form dinitramide.

Due to the relatively high freezing point of concentrated sulphuric acid, the viscosity of the mixture will quickly increase as the temperature decrease. Addition of nitric acid decrease the viscosity but controlling temperature and the flow of nitric acid was found to be crucial due to the exothermal reaction. At too high temperature the dinitramide formed will decompose, and if the temperature or the flow of nitric acid is too low the slurry will freeze and the system will halt.

Another challenge was the pumping of the reaction slurry. Solenoid pumps are not suitable for this type of feeding due to the air bubbles in the slurry which influence the vacuum needed to drive the flow. Using a peristaltic pump eliminated this problem but peristaltic pumps generate less pressure then the solenoid pumps.

The development of a continuous nitration method has been more complex than expected. The progress made has been substantial and many technical problems have been solved. It was however in this work not possible to demonstrate a fully functioning continuous nitration method. Nevertheless, due to the progress made, it is considered that continues nitration is the most promising way to produce dinitramide and that the remaining technical challenges can be solved.
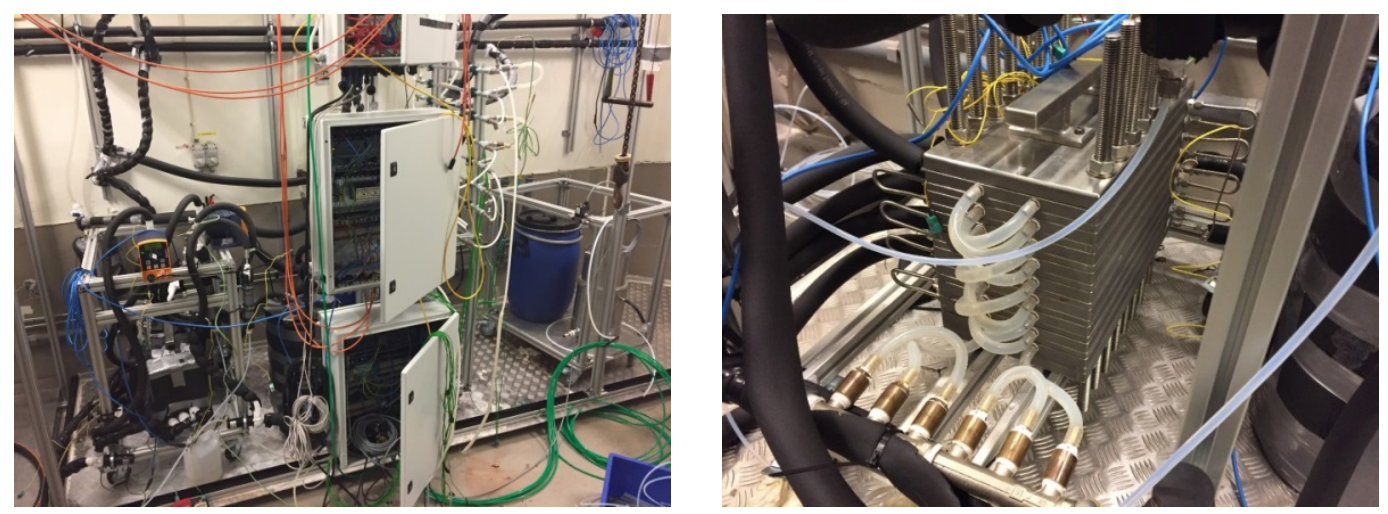

Fig. 6 [Left] Pilot plant for continuous titration. [Right] Continuous flow plate reactor.

\section{Experimental verification of system requirements in $20 \mathrm{~N}$ catalytic thruster demonstrator unit}

Two thruster demonstrators were planned in the Rheform project: a $20 \mathrm{~N}$ thruster with catalytic ignition and a $200 \mathrm{~N}$ thruster with thermal ignition. Due to the problem in developing a working thermal igniter, only the $20 \mathrm{~N}$ thruster with catalytic ignition was finalized.

The thruster was designed and manufactured from the project partner ECAPS. It was designed to facilitate the loading of the catalysts, so giving the possibility to test with different catalysts. A picture of the thruster is shown in Fig. 7. An important part of the manufacturing process was the construction of the reaction chamber where the heat bed and catalyst resides. The material used for this has been molybdenum. As this type of material has got a long procurement time it was ordered early in the design process. Due to time limitation only a catalyst was tested in the thruster: Ba-Ir-Co-Al hexaluminate pellets synthetized by CNRS. 


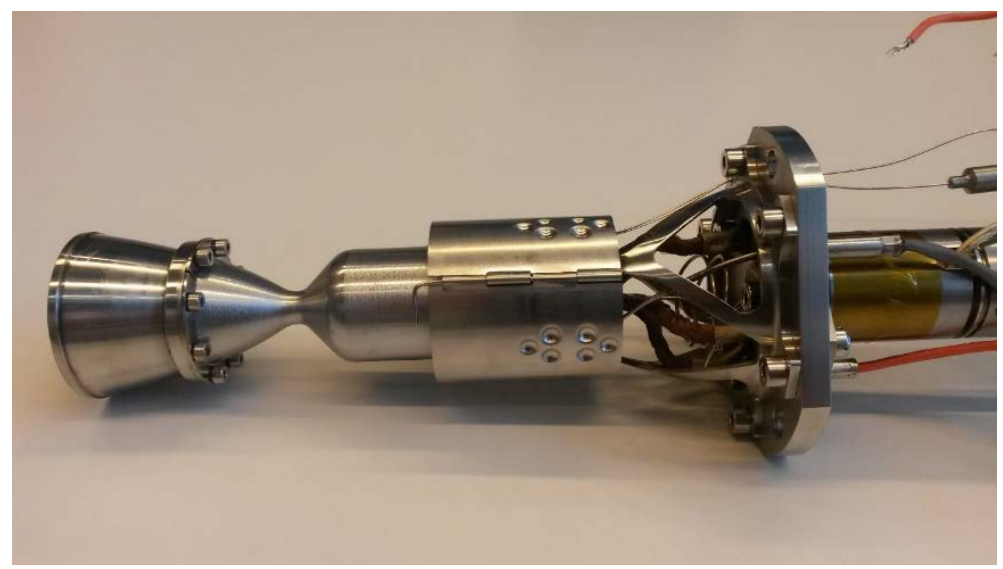

Fig. $720 \mathrm{~N}$ catalytic thruster demonstrator.

The catalyst was loaded in the thruster at ECAPS in Sweden. There also pre-tests (leakage, electronics...) were conducted. Subsequently the thruster was shipped to DLR, for hot firing.

The hot firing tests were conducted at the test bench M11.2 in Lampoldshausen. The thruster was mounted in a $4 \mathrm{~m}^{3}$ chamber which was evacuated to simulate high altitude conditions. Two mechanical pumps were used, which allowed reaching a pressure of 3 mbar in the chamber before ignition. More details on the test bench are given in [8]. The thruster was mounted on a thrust balance. The thrust balance was calibrated by loading it with two different weights, corresponding to the minimum and the maximum expected thrust. The propellant tank was suspended on a scale to measure the amount of propellant injected during each firing. The thruster was equipped with several thermocouples. The most significant thermocouple was a type $\mathrm{R}$ one located in the reactor, and measured the preheating temperature as well as the temperature evolution when propellant was injected. The FCV was a series redundant, dual coil, dual seat, and it was controlled with 28 VDC. The thruster was equipped with $50 \mathrm{~W}$ heater.

14 hot firing tests were conducted. In each test the FCV was opened in a single pulse from 20 to $50 \mathrm{~ms}$ long. The pre-heating temperature was $350^{\circ} \mathrm{C}$, except in the last tests, where it was increased to $410^{\circ} \mathrm{C}$. The thrust measured corresponded to that expected from a vaporization of the propellant, without ignition. The temperature measured in the heater dropped following the firing. It was concluded that the catalyst is not active enough to assure the ignition of the propellant in the thruster tested.

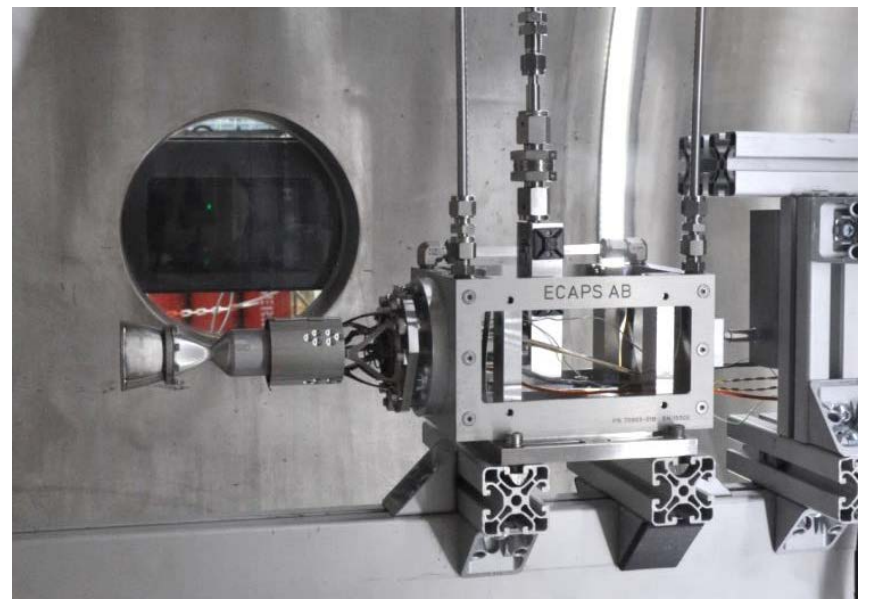

Fig. 8 Thruster and thrust balance mounted in the vacuum chamber for hot firing testing. 


\section{Adaptation of numerical models used for catalytic ignition systems and of models used for thermal ignition systems}

The project partner FOTEC has developed an in-house code to simulate the processes happening in the catalyst bed. During the Rheform project the code was adapted such that catalyst structures with arbitrary geometry can be imported from CAD-files. This includes the automatic detection of cross-sectional shape and area by the code. As an arbitrary catalyst structure is not necessarily symmetric around its longitudinal axis, simulations were performed on the whole catalyst and surrounding structure. This resulted in a significant increase in computational costs.

In addition a simple model was developed to predict the probability the carrier substrate fails due to thermal shock. Whereas failure of ductile materials, such as metals, is largely determined by the plastic behaviour of the material, the failure of brittle materials is mainly dependent on the crack initiation process. To predict the likelihood of failure of ceramics, use was made of the Weibull weakest link theory. The probability density function is dependent on the number of materials flaws present per unit volume. The theory is applicable to single load cases as well as cyclic loads. Its application is limited by the availability of material properties.

\section{Obtaining experimental data for further validation of numerical models}

A series of monolithic catalysts with varying internal geometries was designed to understand the influence of the internal structure on the performance. The internal geometry design of these catalysts was such that the catalytic surface area to solid volume ratio and Reynolds number was the same. The catalytic surface area to solid volume ratio is an important figure of merit of the temperature response of the catalyst as has become clear during the first set of catalyst pre-selection testing. Reynolds number similarity on the other hand ensures a similar level of turbulence in the flow. Note that this does not mean that the pressure drop over the catalyst is the same. This is due to the two-phase flow rather than a single phase flow. The friction between each phase and the solid is similar for equal Reynolds number. A difference in measured pressure drop over the catalyst is therefore an important indication of the effectiveness of the catalyst as the majority of the pressure drop is in the gas phase.
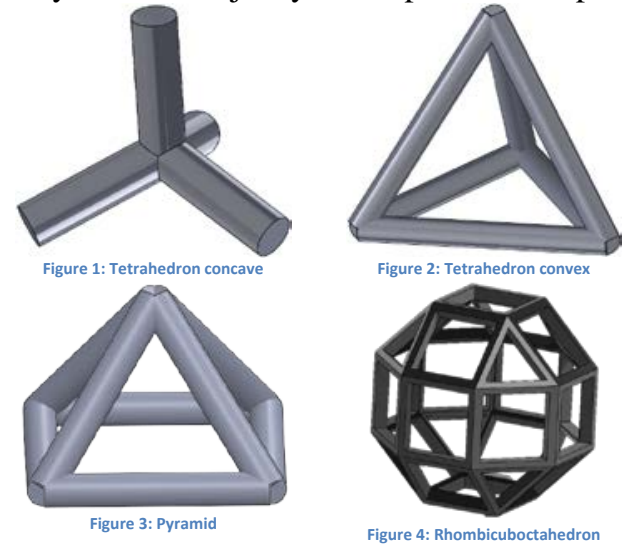

Fig. 9 Geometries considered when analyzing the influence of the monoliths internal structure on performance.

\section{Conclusion}

In the European project Rheform research activities aimed at improving ADN-based propulsion systems have been conducted. Rheform is a project funded from the European's Union Horizon 2020 programme. The name Rheform stands for: "Replacement of hydrazine for orbital and launcher propulsion systems". The project ran from January 2015 to the end of 2017. In the present paper an overview of the main results is given. 


\section{Acknowledgments}

This project has received funding from the European Union's Horizon 2020 research and innovation programme under grant agreement No 640376 .

The authors would like to thank the M11 team for the help during test conduction.

\section{References}

[1] A. S. Gohardani et al., "Green space propulsion: Opportunities and prospects," Prog. Aerosp. Sci., vol. 71, pp. 128-149, Nov. 2014.

[2] K. Anflo and B. Crowe, "In-Space Demonstration of High Performance Green Propulsion and its Impact on Small Satellites," presented at the 25th Conference on Small Satellites, 2011.

[3] M. Negri et al., "New technologies for ammonium dinitramide based monopropellant thrusters - The project RHEFORM,” Acta Astronaut., vol. 143, pp. 105-117, Feb. 2018.

[4] S. A. Whitmore, D. P. Merkley, S. D. Eilers, and T. L. Taylor, "Hydrocarbon-Seeded Ignition System for Small Spacecraft Thrusters Using Ionic Liquid Propellants," presented at the 27th Conference on Small Satellites.

[5] Aerojet, "Monopropellant Thrusters Data Sheet," 2006. [Online]. Available: https://www.rocket.com/files/aerojet/documents/Capabilities/PDFs/Monopropellant Data Sheets.pdf.

[6] M. Negri, M. Wilhelm, and C. Hendrich, "Thermal ignition of ADN-based propellants," presented at the Space Propulsion Conference, Seville, Spain, 2018.

[7] “GRAIL Website.” [Online]. Available: http://grail-h2020.eu/.

[8] M. Wilhelm, C. Hendrich, H. Zimmermann, H. Ciezki, and S. Schlechtriem, "Test Facility for Research on Advanced Green Propellants under High-Altitude Conditions,” presented at the Space Propulsion Conference, 2018, p. 17. 